\title{
The Influence of Electrode Material of Planar Capacitor on Magnetoelectric Properties in Ferromagnetic Oxide Sputtered Films with the $\mathrm{Bi}_{2} \mathrm{O}_{3}-\mathrm{Fe}_{2} \mathrm{O}_{3}-\mathrm{PbTiO}_{3}$ System
}

\author{
T. Higashi, A. Kajima, M. Inoue* and T. Fujii** \\ Kitakyushu National College of Technology, 5-20-1 Shii, Kokuraminami-ku, Kitakyushu 802-0985, Japan \\ *Toyohashi University of Technology, 1-1 Hibarigaoka Tenpaku-cho Toyohashi 441-8580, Japan \\ ** Professor Emeritus, Toyohashi University of Technology
}

A magnetoelectric (ME-) interaction such a multiferroic property as the dielectric permittivity $\varepsilon_{r}^{\prime}$ (the real part of the complex permittivity) is changed by magnetic field $H, \Delta \varepsilon_{\mathrm{r}}{ }^{\prime}(H)$, was studied in nano-composite oxide films of the $\mathrm{Bi}_{2} \mathrm{O}_{3}-\mathrm{Fe}_{2} \mathrm{O}_{3}-\mathrm{PbTiO}_{3}$ system prepared by rf-reactive sputtering. In our past studies, vacuum-evaporated $\mathrm{Al}$ film was deposited on the film surface to form the planar capacitor to measure the dielectric properties. However, the temporal change of $\Delta \varepsilon_{r}^{\prime}(H)$, was occasionally observed. In the present study, we replaced Al-film with sputtered In-Sn-O (ITO) film and succeeded to reduce greatly the thermal instability. Thus we can obtain more precise and reliable data of $\Delta \varepsilon_{r}^{\prime}(H)$, which enables us to elucidate the mechanism of this phenomenon.

Key words: magnetoelectric effect, magnetic field sensitive dielectric permittivity, ferromagnetic oxide film, reactive rf-sputtering

\section{Introduction}

We have previously reported that the real part of the dielectric complex permittivity, $\varepsilon_{\mathrm{r}}$ ' (i.e. $\varepsilon_{\mathrm{r}}=\varepsilon_{\mathrm{r}}$ ' $-j \varepsilon_{\mathrm{r}}$ ") of ferromagnetic nano-composite oxide sputtered films with the $\mathrm{Bi}_{2} \mathrm{O}_{3}-\mathrm{Fe}_{2} \mathrm{O}_{3}-\mathrm{PbTiO}_{3}$ ternary system is subject to change with an external magnetic field $H$ at room temperature, and its maximum of the relative change of er' with $H$, i.e. $\left|\Delta \varepsilon_{\mathrm{r}}{ }^{\prime}(H) / \varepsilon_{\mathrm{r}}{ }^{\prime}\right| \max$ reached as large as $0.12 \%$ in a wide frequency range from $1 \mathrm{~Hz}$ to $100 \mathrm{kHz}^{1}$. $\Delta \varepsilon_{\mathrm{r}}{ }^{\prime}(H)$-curve of the film shows hysteresis loop, which is closely correlated with its magnetization curve. $\left.{ }^{2}\right)$

In recent years, other type of oxides that exhibit the magnetocapacitance effect have been reported, e.g., $\mathrm{BiMnO}_{3}$ polycrystal ${ }^{3)}$ and $\mathrm{BaTiO}_{3}-\mathrm{CoFe}_{2} \mathrm{O}_{4}$ nanocomposite $^{4)}$. In the former, the capacitance change with $H, \Delta C(H)$ was observed below $130 \mathrm{~K}$ to keep the magnetic ordering. In the latter, $\Delta C(H)$ was observed at room temperature, but there is no direct correlation between $\Delta C(H)$-curve and its magnetization curve.

We also observed the dielectric polarization induced by applying a small AC magnetic field $h(\omega)$, $\Delta P_{\mathrm{h}}(\omega)$. This is considered to be a new type of magnetoelectric effect ${ }^{5)}$. The detected $\Delta P_{\mathrm{h}}(\omega)$ signal changes depending on both the direction of the magnetic field and the DC bias voltage applied perpendicular to the film plane. To explain these experimental results, we proposed a model based on magnetization rotation of ferromagnetic nano-clusters dispersed in a dielectric glassy matrix ${ }^{5-6}$.

In our past studies, a vacuum-evaporated $\mathrm{Al}$ film was used for the upper electrode of the planar capacitor to measure the dielectric properties of the film. However, the shape of $\Delta \varepsilon_{\mathrm{r}}{ }^{\prime}(H)$-curve is occasionally changed with ageing time even the film is kept at a low temperature as $50{ }^{\circ} \mathrm{C}$. In the present study, we replaced an $\mathrm{Al}$-film with an In-Sn-O (ITO) film and found that such a thermal instability is greatly suppressed, which enables us to obtain the precise and reliable data to elucidate the origin of this property.

\section{Experiment}

The sputtering conditions are described in [6]. In this study, magnetic films were fabricated with a fixed composition of $0.18 \mathrm{Bi}_{2} \mathrm{O}_{3}-0.70 \mathrm{Fe}_{2} \mathrm{O}_{3}-0.12 \mathrm{PbTiO}_{3}$ (in atomic fraction) because this composition attains the strongest ferromagnetism in the present ternary oxide system. Films about $0.5 \mu \mathrm{m}$ thick were deposited on heavily doped $\mathrm{n}$-type Si-wafers $(\rho=0.0016 \Omega \mathrm{cm})$ to avoid the influence of a depletion layer formed on the film-Si interface ${ }^{7)}$, and subjected to annealing in air at various temperatures. $\varepsilon_{\mathrm{r}}$ was measured by the

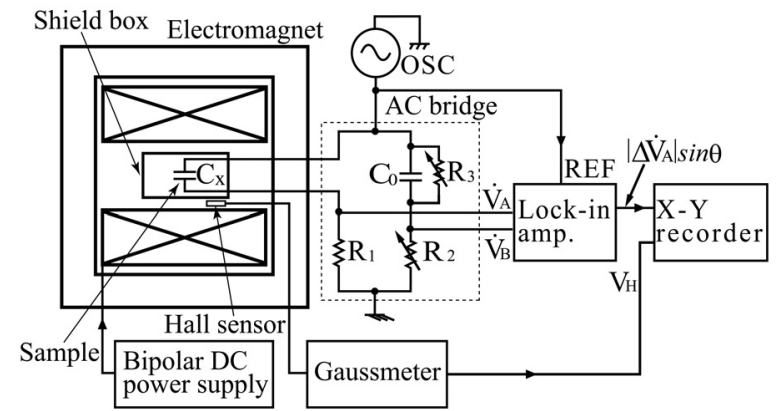

Fig. 1 Circuit diagram for measurement of dielectric permittivity change with magnetic field $\Delta \varepsilon_{\mathrm{r}}{ }^{\prime}(H)$ 
capacitance of a planar capacitor which deposits either vacuum- evaporated Al-film or rf-sputtered ITO-film on the film surface and an In-film was deposited on the back surface of the low-resistivity Si-substrate to ensure ohmic contact.

$\Delta \varepsilon_{\mathrm{r}}^{\prime}(H)$ was measured by an AC-bridge utilizing lock-in detection and determined as the out-phase component of the output signal with respect to the reference signal; the circuit diagram is drawn in Fig. 1. The planar capacitor of the sample is installed in a shield box set in the air-gap of an electromagnet, connected to the AC-bridge, and kept at a constant temperature during measurement. The $\mathrm{AC}$ electric field $E$ is applied across the film thickness, the drive frequency $f$ is changed from $1 \mathrm{~Hz}$ to $100 \mathrm{kHz}$, where $\varepsilon$ ' is calculated from the out-phase component of the voltage drop across a resistor $R_{1}$ inserted in series with the planar capacitor. After the AC-bridge is balanced, $H$ up to $\pm 10 \mathrm{kOe}$ is applied parallel to the film plane.

\section{Results}

\subsection{Film structure}

Figure 2 is the change of XRD patterns of a film by successive post-annealing. In as-sputtered state, XRD shows a halo-pattern, indicating that the sample is amorphous. With elevating annealing temperature, $T_{\mathrm{a}}$, faint diffraction signals assigned to $\mathrm{BiFeO}_{3}-\mathrm{PbTiO}_{3}$ perovskite solid solutions, $\mathrm{Bi}_{2} \mathrm{Fe}_{4} \mathrm{O}_{9}$ and $\alpha-\mathrm{Fe}_{2} \mathrm{O}_{3}$ appear. However, these materials are not ferromagnetic and considered to be precipitated in the vicinity of the film surface and ferromagnetism arises from the nanocomposite part inside the film ${ }^{8)}$.

\subsection{Influence of electrode material}

The series of $\Delta \varepsilon_{\mathrm{r}}{ }^{\prime}(H)$-curves depicted in Fig. 3 are the variation of $\Delta \varepsilon_{\mathrm{r}}{ }^{\prime}(H)$ of a sample with an Al-electrode

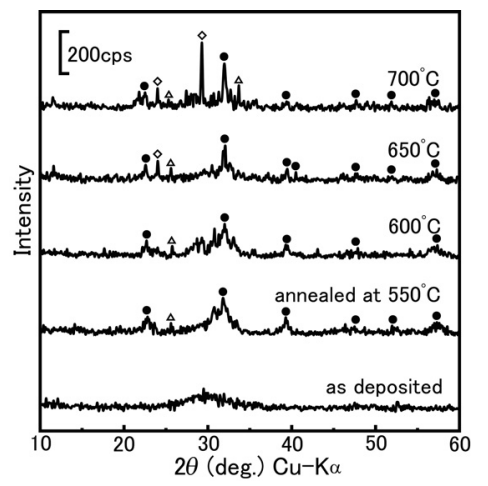

Fig. 2 XRD patterns for various annealing temperatures. The diffraction lines indicated by various marks are assigned to $\mathrm{O} \mathrm{BiFeO}_{3}{ }^{-}$ $\mathrm{PbTiO}_{3}$ perovskite solid solutions, $\triangle: \alpha-\mathrm{Fe}_{2} \mathrm{O}_{3}$, $\diamond: \mathrm{Bi}_{2} \mathrm{Fe}_{4} \mathrm{O}_{9}$.

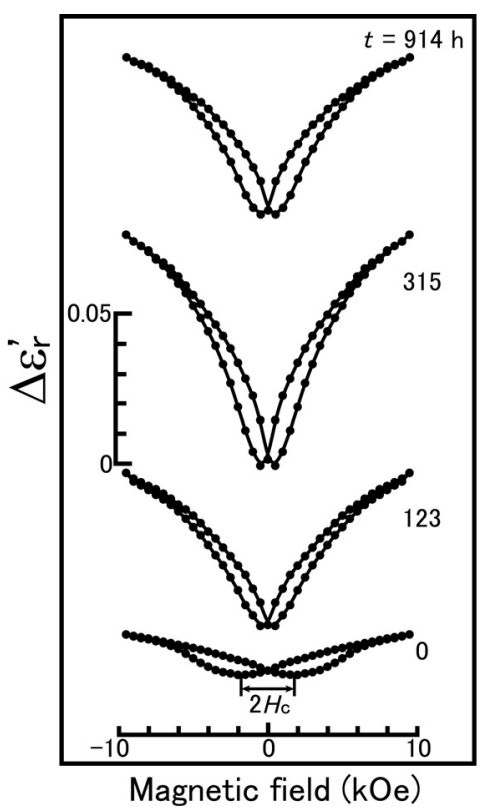

Fig. 3 The temporal change of $\Delta \varepsilon_{\mathrm{r}}{ }^{\prime}(H)$-curve for a film with Al-electrode. The film was preannealed at $600{ }^{\circ} \mathrm{C}$ for $3 \mathrm{~h}$. The ambient temperature during measurement is $50{ }^{\circ} \mathrm{C}, f=$ $1 \mathrm{kHz}$.

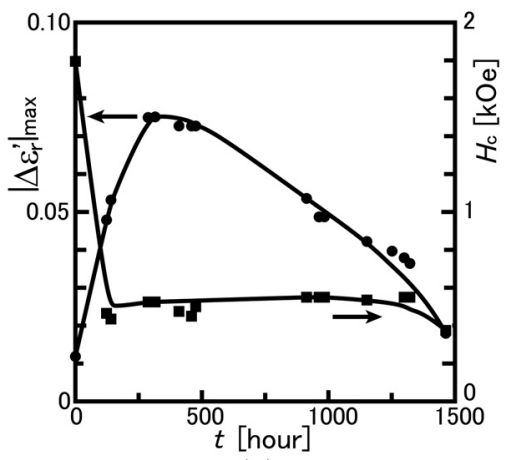

(a)

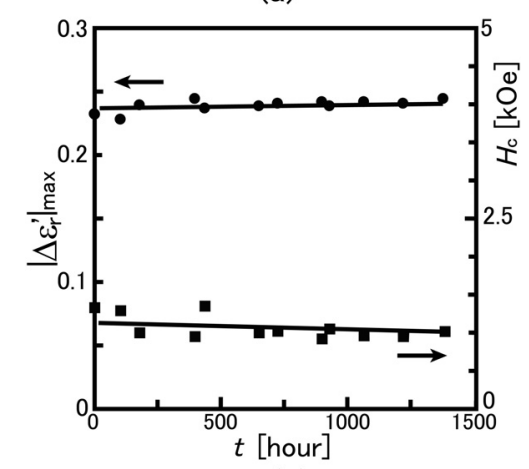

(b)

Fig. 4 The temporal change of $\Delta \varepsilon_{\mathrm{r}}^{\prime}(H)$-curve for a film with Al-electrode. The film was pre-annealed at $600{ }^{\circ} \mathrm{C}$ for $3 \mathrm{~h}$. The ambient temperature during measurement is $50{ }^{\circ} \mathrm{C}, f=$ $1 \mathrm{kHz}$. 
for some ageing times $t$, where the ageing temperature was kept at $50{ }^{\circ} \mathrm{C}, f=1 \mathrm{kHz}$ and the sample was preannealed at $600{ }^{\circ} \mathrm{C}$ for 3 hours. Notwithstanding the sample was kept at such low temperature as $50{ }^{\circ} \mathrm{C}$, a drastic change of $\Delta \varepsilon_{\mathrm{r}}{ }^{\prime}(H)$ takes place with the elapse of time. From Fig.3, $\left|\Delta \varepsilon_{\mathrm{r}}(H)\right|_{\max }$ and $H_{\mathrm{c}}$ (which is determined by $H$, at which $\left|\Delta \varepsilon_{\mathrm{r}}{ }^{\prime}(H)\right| \max$ takes minimum) are plotted against $t$ in Fig. 4(a). Both quantities vary in a complicate way with $t$. On the contrary, Fig. 4(b) is the same plot for a sample with an ITO-electrode. In this case, no remarkable changes in both quantities are observed, indicating that thermal stability is improved. These facts suggest that the film structure was changed by the diffusion of $\mathrm{Al}$ into film. Henceforth material of the upper electrode was replaced from Al to ITO to obtain more reliable data of the magnetoelectric properties.

\subsection{ME-properties of the film with ITO electrode}

Figure 5 shows the temperature dependence of $\Delta \varepsilon_{\mathrm{r}}{ }^{\prime}(H)$ of a sample annealed at $600^{\circ} \mathrm{C}$ for 3 hours $(f=1$

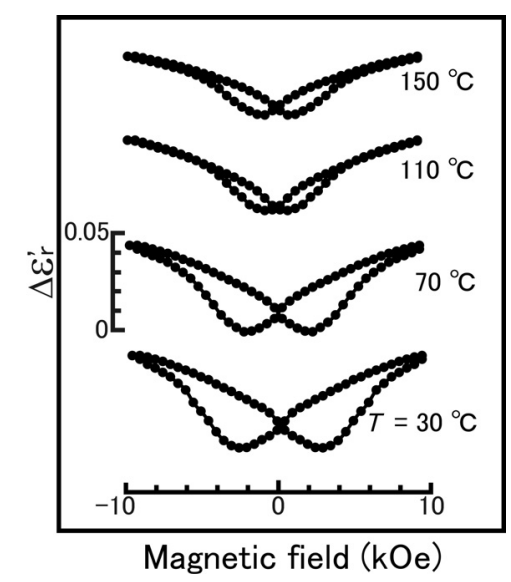

Fig. 5 The temperature dependence of $\Delta \varepsilon_{\mathrm{r}}{ }^{\prime}(H)$-curve for a sample with ITO-electrode. The sample was pre-annealed at $600^{\circ} \mathrm{C}$ for $3 \mathrm{~h}, f=1 \mathrm{kHz}$.

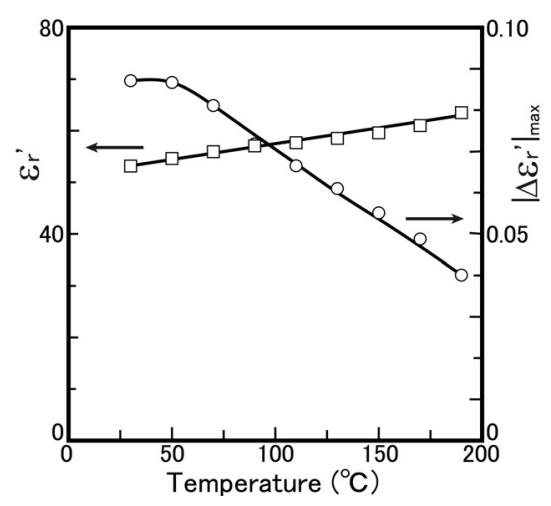

Fig. $6 \varepsilon_{r}^{\prime}$ and $\left|\Delta \varepsilon_{r}^{\prime}(H)\right| \max$ vs. temperature for the sample shown in Fig. 4.
$\mathrm{kHz}) ;\left|\Delta \varepsilon_{\mathrm{r}}^{\mathrm{r}}(H)\right|_{\max }$ and $H_{\mathrm{c}}$ decreased with rising temperature T. $\varepsilon_{\mathrm{r}}^{\prime}$ and $\left|\Delta \varepsilon_{\mathrm{r}}^{\prime}(H)\right| \max$ are plotted as a function of temperature in Fig. 6, and a negative correlation between both dielectric quantities is found at least in this temperature range. In the previous study, it was shown that the saturation magnetization, $4 \pi M_{\mathrm{s}}$ of the film with same composition decreased monotonically with temperature ${ }^{7)}$. In fact, $\Delta \varepsilon_{\mathrm{r}}{ }^{\prime}(H)$ is proportional to $\{4 \pi M(H)\}^{2} 2$.

Figure 7 shows that $\Delta \varepsilon_{\mathrm{r}}^{\prime}(H)$-curve changes significantly with elevating annealing temperature $T_{\text {a }}$. Both of $\varepsilon_{\mathrm{r}}^{\prime}$ and $\left|\Delta \varepsilon_{\mathrm{r}}{ }^{\prime}(H)\right|_{\max }$ decrease rapidly above $T_{\mathrm{a}}=$ $650^{\circ} \mathrm{C}$ (notice that the scale of $\Delta \varepsilon_{\mathrm{r}}{ }^{\prime}(H)$ of $T_{\mathrm{a}}=650^{\circ} \mathrm{C}$ and $700{ }^{\circ} \mathrm{C}$ is magnified by 50 !). These values are listed in Table 1.

Magnetic properties of the sample are measured with a VSM. Figure 8 shows the room-temperature magnetization curves of the same film used in Fig. 7, where $H$ was applied parallel to the film plane. $4 \pi M_{\mathrm{s}}$ was estimated from magnetization at $H=10 \mathrm{kOe}$, which is the maximum field available for our VSM. $H_{\mathrm{c}}$ of each sample is exactly coincided with $H$ at which $\Delta \varepsilon_{\mathrm{r}}{ }^{\prime}(H)$ takes minimum. In contrast with $\Delta \varepsilon_{\mathrm{r}}{ }^{\prime}(H), 4 \pi M_{\mathrm{s}}$ and $H_{\mathrm{c}}$ continue to increase with $T_{\mathrm{a}}$.

To explain the experimental results, we previously

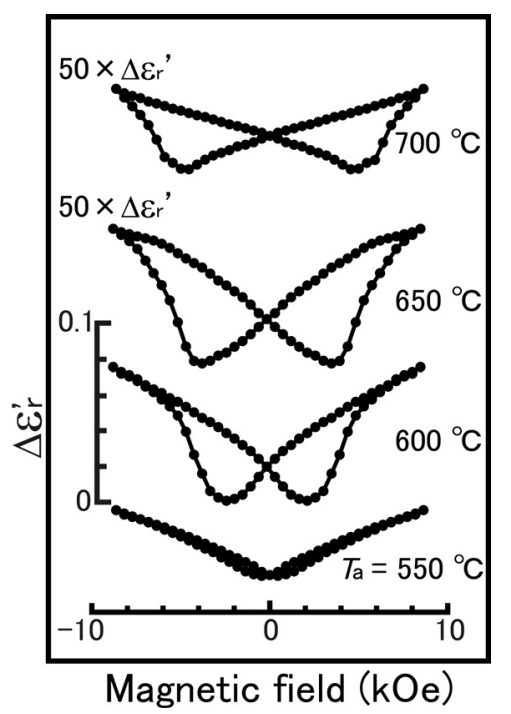

Fig. 7 Variation of $\Delta \varepsilon_{\mathrm{r}}(H)$-curve by pre-annealing for $3 \mathrm{~h}$. The ambient temperature is $50^{\circ} \mathrm{C}, f=1 \mathrm{kHz}$.

Table $1 \varepsilon_{\mathrm{r}}{ }^{\prime}$ and $\left|\Delta \varepsilon_{\mathrm{r}}{ }^{\prime}(H)\right|_{\max }$ of the films used in Fig. 7.

\begin{tabular}{c|c|c}
\hline$T_{\mathrm{a}}\left({ }^{\circ} \mathrm{C}\right)$ & $\varepsilon_{\mathrm{r}}{ }^{\prime}$ & $\begin{array}{c}\left|\Delta \varepsilon_{\mathrm{r}}{ }^{\prime}(H)\right|_{\mathrm{ma}} \\
\mathrm{x} \\
\left(10^{-3}\right)\end{array}$ \\
\hline 550 & 65.09 & 28.29 \\
\hline 600 & 42.40 & 57.43 \\
\hline 650 & 6.51 & 1.16 \\
\hline 700 & 5.47 & 0.71 \\
\hline
\end{tabular}




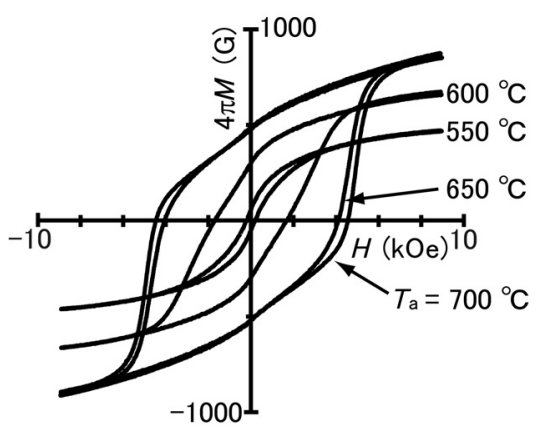

Fig. 8 Room-temperature magnetization curves of the films used in Fig. 7.

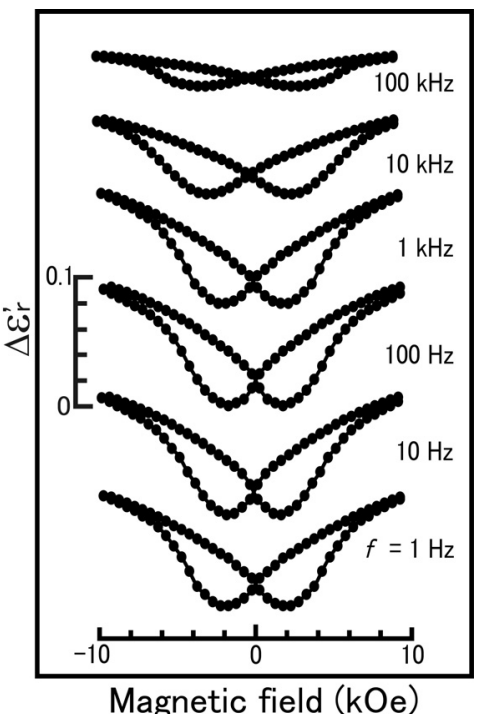

Fig. 9 The variation of $\Delta \varepsilon_{\mathrm{r}}^{\prime}(H)$-curve with frequency $f$. The sample was pre-annealed at $600{ }^{\circ} \mathrm{C}$ for 3 $\mathrm{h}$. The temperature during measurement is $50{ }^{\circ} \mathrm{C}$.

proposed a simple model based on magnetization rotation of ferromagnetic nano-sized clusters randomly dispersed in the dielectric glassy matrix. The magnetostatic energy among the clusters is stored in dielectric glassy matrix. The deformation of the matrix network caused by, for example, magnetostriction of the clusters by applying $H$ gives rise to the appearance of $\Delta \varepsilon_{\mathrm{r}}{ }^{\prime}(H)$. Therefore the results shown in Figs. 7 and 8 suggest that heat treatment above $650{ }^{\circ} \mathrm{C}$ reinforces the magnetic moment of the cluster, while inactivates the dielectric moment of the matrix.

Figure 9 shows $\Delta \varepsilon_{\mathrm{r}}^{\prime}(H)$-curve of a sample at different frequencies. $\left|\Delta \varepsilon_{\mathrm{r}}(H)\right|_{\max }$ is decreased with $f$ and decrease rapidly above $f=10 \mathrm{kHz}$. It still remains a doubt that the rapid decrease of $\Delta \varepsilon_{\mathrm{r}}{ }^{\prime}(H)$ with $f$ is superficial caused by the increase of line impedance between the sample capacitor and AC-bridge. We now check the measurement system.

\section{Concluding Remarks}

We have investigated the magnetoelectric (ME-) effect of ferromagnetic oxide films of the $\mathrm{Bi}_{2} \mathrm{O}_{3}$ $\mathrm{Fe}_{2} \mathrm{O}_{3}-\mathrm{PbTiO}_{3}$ system prepared by rf-reactive sputtering. The measurement was done by the dielectric permittivity change with magnetic field, $\Delta \varepsilon_{\mathrm{r}}{ }^{\prime}(H)$, for films formed in planar capacitor. Two different materials were used as the upper electrode of the capacitor; vacuum-evaporated $\mathrm{Al}$ film and sputtered ITO-film. Drastic and complicated changes in $\Delta \varepsilon_{\mathrm{r}}{ }^{\prime}(H)$ are observed with the elapse of time even at low temperature in films with Al-electrode, while no remarkable change occurs in films with ITO-electrode. Thus ITO-electrode is found to be adequate for elucidation of this phenomenon. The temporal instability of Al-electrode may be caused by penetration of Al-atoms into the film by thermal diffusion and $\mathrm{Al}$-atoms are combined with oxygen atoms in the film to form oxides such as $\mathrm{Al}_{2} \mathrm{O}_{3}{ }^{9}{ }^{9}$ Microscopic observation such as XPS, FE-SEM, etc. is necessary for making clear these facts, which is now in progress. By the way, films with ITO-electrode have a high optical transmittance in the visible region, so we are now planning to try the optical probing of this phenomenon utilizing magneto- (and/or electro-) optical effects.

\section{Acknowledgments}

The authors are indebted to Messrs. T. Imazawa, T. Hanada, H. Sakouchi, R. Nishimoto, S. Miyashita, T. Aoyagi, H. Kuboi, Y. Sueyoshi, A. Masuda, and R. Yamada for their experimental assistances throughout the investigation.

\section{References}

1) A. Kajima, Y. Nakamura, M. Inoue, and T. Fujii: J. Magn. Soc. Jpn., 25, 875 (2001) [in Japanese].

2) A. Kajima, R. Nakayama, M. Inoue, and T. Fujii: J. Magn. Soc. Jpn., 26, 445 (2002) [in Japanese].

3) T. Kimura, S. Kawamoto, I. Yamada, M. Azuma, M. Takano and Y. Tokuda: Phys. Rev. B, 67, 180401 (2003).

4) I. Fina, N. Dix, L. Fàbrega, F. Sánchez, and J. Fontcuberta: Thin Solid Films, 518, 4634 (2010).

5) A. Kajima, R. Nakayama, T. Ohashi, M. Inoue, and T. Fujii: J. Magn. Soc. Jpn., 27, 348 (2003) [in Japanese].

6) A. Kajima, T. Ohashi, M. Inoue, and T. Fujii: J. Magn. Soc. Jpn., 28, 981 (2004) [in Japanese].

7) A. Kajima, T. Arita, Y. Tsuji, M. Inoue, and T. Fujii: J. Magn. Soc. Jpn., 30, 174 (2006) [in Japanese].

8) A. Kajima, T. Kaneda, H. Ito, T. Fujii, and I. Okamoto: $J$. Appl. Phys., 70, 3760 (1991).

9) M. He and T. M. Lu: Metal-Dielectric Interface in Gigascale Electronics, Thermal and Electrical Stability, p.18 (Springer, New York, 2012)

Received October 18, 2012; Revised December 11, 2012; Accepted January 7, 2013 